\title{
ONCOPLASTIC SURGERY IN BREAST- CONSERVATIVE TREATMENT: PATIENT PROFILE AND IMPACT ON QUALITY OF LIFE
}

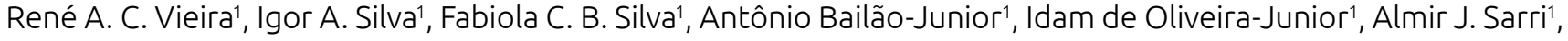
Jonathas J. Silva', Carlos E. Paiva'

${ }^{1}$ Barretos Cancer Hospital, Pio XII Foundation - Barretos (SP), Brazil.

Background: Breast conserving-surgery (BCS) patients have better quality of life (QL) than mastectomy without reconstruction. Oncoplastic surgery (OS) is becoming increasingly common, but few studies have evaluated the impact on QL in patients submitted BCS with OS. Objective: Evaluate the quality of life in patients submitted to BCS with OS. Methods: Ethics Committee approval 782/2014. A prospective, cross-sectional, controlled study that was conducted with 300 women who were submitted to BCS. QL was assessed through the EORTC QLQ-30, EORTC QLQ-BR23, and BCTOS questionnaires. We evaluated the characteristics of patients who underwent BCS with OS and QL. Cosmesis was evaluated by BCCT.core. In the comparison of categorical variables, the chi-square test was used, whereas continuous variables were assessed using the Mann-Whitney test. Results: A total of 300 patients underwent CBT, 72 underwent breast OS, where 37 underwent bilateral surgery. Patients who were submitted to OS were younger $(\mathrm{p}=0.004)$, had a higher level of education $(\mathrm{p}=0.01)$, a smaller time interval since the end of treatment ( $\mathrm{p}=0.02)$, more advanced TNM clinical stage $(\mathrm{p}=0.05)$, tumours with greater dimensions $(\mathrm{p}=0.003)$, were more likely to have T-TNM tumours $(\mathrm{p}=0.02)$ and were more likely to receive neoadjuvant chemotherapy ( $\mathrm{p}=0.05$ ). Evaluating QL questionnaires no difference was observed in the groups. Breast symmetry was not associated with higher patient satisfaction $(\mathrm{p}=0.55)$. Conclusions: A selection bias may have occurred in selecting patients for OS. In our patients, the simple use of OS, whether it was associated with symmetrisation or not, was not associated with improvement in QL. 